\title{
基于不同取向的 CCD 图像准确求解底片常数模型
}

\author{
彭青玉 ${ }^{(12)(3)}$, 范良艳 1 (12) \\ (1) 暨南大学计算机科学系, 广州 510632; \\ (2) 广东省高等学校光电信息与传感技术重点实验室(暨南大学), 广州 510632; \\ (3) 中国科学院光学天文联合实验室, 昆明 650011 \\ E-mail: pengqy@pub.guangzhou.gd.cn \\ 2009-07-10 收稿, 2009-09-28 接受 \\ 国家自然科学基金和中国科学院天文联合基金(批准号: 10573008, 10778617, 10973007)资助项目
}

\begin{abstract}
摘要 使用云南天文台 1 米望远镜上新安装的 $2 k \times 2 k \mathrm{CCD}$ 观测疏散星团 NGC2168, 并在观测 过程中旋转 CCD 支架 $90^{\circ}$ 分别成像. 采用 UCAC2 星表的星表位置对视场中星象的量度坐标进 行归算表明: CCD 视场具有明显的扭曲, 即在星象标准坐标 $(\xi, \eta)$ 与量度坐标 $(x, y)$ 的关系模型中, $(x, y)$ 对 $(\xi, \eta)$ 的影响高达 4 次方项. 另一方面, 较亮的 UCAC2 星的位置测量精度可达 7 mas(视 场大小为 $7^{\prime} \times 7^{\prime}$, 曝光时间为 $60 \mathrm{~s}$ ). 在高精度定位的 CCD 成像观测中, 这一新的发现对于提高 天体测量精度将具有重要的意义.
\end{abstract}

\section{关键词}

$\mathrm{CCD}$ 成像

视场扭曲

位置测量

图像分析
因为哈勃空间望远镜图像的欠采样, 长期困扰 高精度的天体测量工作. 然而, 自 Anderson 和 King 提出有效点扩散函数 (ePSF) 概念 ${ }^{[1]}$ 、实施高精度 $\mathrm{ePSF}$ 拟合并精确求解视场的扭曲 ${ }^{[2]}$ 以后, HST 的天体测量 工作才有了实质性的飞跃. 例如土星卫星的观测, 在 精确求解 HST 视场扭曲以前, 行星照相机(PC)和大 视场照相机(WF) 观测主要卫星(S1-S6)的位置精度 ${ }^{[3]}$ 分别为 $0 .{ }^{\prime \prime} 04$ 和 $0 .{ }^{\prime \prime} 07$. 然而, French 等人 ${ }^{[4]}$ 利用 Anderson 和 King 精确求解视场扭曲结果后, 主要卫星的位 置测量精度提高到 0." $01(\mathrm{PC})$ 和 0." $04(\mathrm{WF})$ ，应该注意， HST 的小视场(对 PC 和 WF, 其视场大小分别为 $36^{\prime \prime}$ $\times 36^{\prime \prime}$ 和 $\left.80^{\prime \prime} \times 80^{\prime \prime}\right)$ 内扭曲的影响高达量度坐标 $(x, y)$ 的 3 次方项! 对于地基望远镜的观测者, 这是难以理解 的. 由 HST 的观测资料能探测到高阶扭曲项关键的 原因在于采用 ePSF 技术后, 星象量度坐标测量精度 的提高. 为什么这样小视场会存在如此高阶项扭曲 的本质, Anderson 和 King ${ }^{[2]}$ 认为, 天文仪器的设计者 非常倾向于得到锐利的星象, 而很少或从不在乎它 们成像的位置! 地面长焦距望远镜 CCD 图像通常不 存在欠采样, 但人们很少关心视场的扭曲. 例如, 在
地面望远镜观测土星卫星的资料归算中, 人们常常 采用 4 常数模型 ${ }^{[58]}$ 求解 $\mathrm{CCD}$ 图像的比例尺(scale)和 取向 (orientation)。这是最简单的归算模型，而且土 星主要卫星的位置测量精度不如 HST 的测量精度(卫 星之间互掩互食的观测除外), 最好精度为 $0 .{ }^{\prime \prime} 04$. 当然, 测量精度不高的原因也包括行星晕的影响, 有 时候甚至还是严重的影响 ${ }^{[9,10]}$. 对于木星和土星主要 卫星的观测, 卫星之间角距离通常不是很小(例如土 卫六与土星中心平均冲时的角距离可达 3.'3, 木卫 四与木星中心的角距离最大可达 10.'3), 此时视场 的扭曲影响很可能是不容忽视的. 地面望远镜 CCD 观测时没有很好研究视场扭曲影响的一个原因是, CCD 视场中通常只有少数的几颗卫星, 不能实施高 阶模型的求解.

为了进一步提高长焦距望远镜 $\mathrm{CCD}$ 成像用于行 星卫星的天体测量精度, 2009 年 2 月 25 日晚我们在 中国科学院云南天文台 1 米望远镜上采用 Johnson I 型滤光片在天顶附近尝试观测密集星场. 为了研究 视场的扭曲, 观测过程中我们转动 $\mathrm{CCD}$ 支架 $90^{\circ}$ 分别 成像, 并使用 UCAC2 星表进行归算. 


\section{1 观测资料说明}

2009 年 2 月 25 日晚, 我们使用中国科学院云南 天文台 1 米望远镜新安装的 $2 k \times 2 k \mathrm{CCD}$ 观测了疏散 星团 NGC2168. 望远镜及 CCD 的有关参数见表 1 .

为了得到高精度天体位置的 CCD 成像, 我们在 天顶附近实施跟踪观测. 观测过程中, 我们将 CCD 支架(连同滤光片)一起旋转 $90^{\circ}$ 分别成像. 旋转前我 们观测了 13 幅 CCD 图像, 旋转后得到 14 幅 CCD 图 像. 每幅图像曝光 $60 \mathrm{~s}$. 图 1 为旋转支架前一幅典型 的 $\mathrm{CCD}$ 图像, 其中有圆圈的星象为能从 $\mathrm{UCAC} 2$ 星表 中找到而且旋转 $\mathrm{CCD}$ 支架后在视场也出现的共同星, 计有 62 颗. 图中与圆圈 $\mathrm{S}$ 对应的只是亮星, 而非近 邻的暗星.

\section{2 图像处理和归算}

因为密集星场中单一图像就有上百颗星, 人工 一对一的测量将有很大的工作量. 对此, 我们采用软 件 SExtractor ${ }^{[11]}$ 提取星象, 并获得它们的初始位置. 该软件采用了简单的 1 阶矩方法测量星象的位置. 根 据我们的研究 ${ }^{[12]}$, 在经典定心算法中二维高斯拟合 方法对星象的位置测量精度要优于矩方法和中值方 法. 所以, 我们在已知初位置基础上, 开发了自己的 图像处理软件, 对每一星象实施二维高斯函数拟合 方法自动进行精确测量. 此外, 为了将每一星象的量 度坐标与 UCAC2 星表 ${ }^{[13]}$ 中的星相匹配, 我们根据文 献[14]的方法也开发了自己的程序. 总之, 经过上述 图像处理后, 我们能将 UCAC2 星表中可以找到的并 同时出现在每帧 CCD 图像中的星象一一匹配, 并将 有关信息存于统一的文件中.

为了探讨不同底片常数模型对位置测量结果的 影响, 我们尝试采用了 $4,6,12,20$ 和 30 常数模型进 行归算. 对于 30 常数模型中的每一颗星象, 由 $\mathrm{UCAC} 2$ 星表位置计算的标准坐标 $(\xi, \eta)$ 与量度坐标 $(x, y)$ (相对于视场中心做归一化处理)之间, 我们可以 表示为下面的表达形式(如果为 6,12 和 20 常数模型, 则每一表达式中的项数分别为前 3 项、前 6 项和前 10 项):

$$
\left\{\begin{aligned}
\xi= & a_{1}+a_{2} x+a_{3} y+a_{4} x^{2}+a_{5} x y \\
& +a_{6} y^{2}+a_{7} x^{3}+a_{8} x^{2} y+a_{9} x y^{2}+a_{10} y^{3} \\
& +a_{11} x^{4}+a_{12} x^{3} y+a_{13} x^{2} y^{2}+a_{14} x y^{3}+a_{15} y^{4} \\
\eta= & b_{1}+b_{2} x+b_{3} y+b_{4} x^{2}+b_{5} x y+b_{6} y^{2} \\
& +b_{7} x^{3}+b_{8} x^{2} y+b_{9} x y^{2}+b_{10} y^{3}+b_{11} x^{4} \\
& +b_{12} x^{3} y+b_{13} x^{2} y^{2}+b_{14} x y^{3}+b_{15} y^{4} .
\end{aligned}\right.
$$

如果是 4 常数模型, 则有下面更简单的形式:

$$
\left\{\begin{array}{l}
\xi=a_{1}+a_{2} x-b_{2} y \\
\eta=b_{1}+b_{2} x+a_{2} y
\end{array}\right.
$$

其中 $(\xi, \eta)$ 对应于 $\mathrm{CCD}$ 图像曝光中点时刻星象的标准 坐标. 在标准坐标的计算中, 我们考虑了所有天体测 量效应(即在站心位置的基础上考虑了大气折射和中 心投影改正)的影响.

一旦具体阶数的模型确定以后, 对每一帧 CCD 图像中的 UCAC2 星进行最小二乘拟合后可以得到所 有待求参数的估计值, 进而求出同一 CCD 图像中每 颗 UCAC2 星的观测位置与理论位置在赤经、赤纬两 个方向的偏差 $(\mathrm{O}-\mathrm{C})_{x},(\mathrm{O}-\mathrm{C})_{y}$. 对于多幅 $\mathrm{CCD}$ 图像, 同一颗 UCAC2 星就可以求得它的平均值 $(\overline{\mathrm{O}-\mathrm{C}})_{x}$, $(\overline{\mathrm{O}-\mathrm{C}})_{y}$ 及相应的标准差 $\left(\sigma_{x}, \sigma_{y}\right)$. 按通常的意义, 标 准差 $\left(\sigma_{x}, \sigma_{y}\right)$ 反映了星象的位置测量精度.

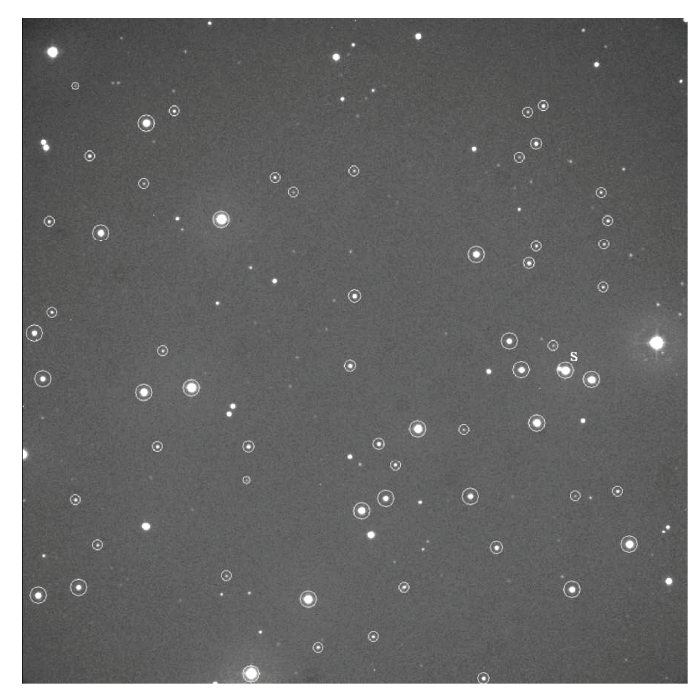

图 1 支架旋转 $90^{\circ}$ 前一幅典型的 CCD 图像

表 11 米望远镜和 CCD 接收器的参数说明

\begin{tabular}{lllllll}
\hline 焦距 & 焦比 & 主镜直径 & CCD 视场 & 像元尺寸 & CCD 阵大小 & 近似比例尺 \\
\hline $1300 \mathrm{~cm}$ & 13 & $100 \mathrm{~cm}$ & $7^{\prime} \times 7^{\prime}$ & $13.5 \mu \times 13.5 \mu$ & $2048 \times 2048$ & $0 .{ }^{\prime 2} 2 / \mathrm{pixel}$ \\
\hline
\end{tabular}




\section{3 位置测量的结果}

由前面的分析可知, 对相同取向的 CCD 图像, 只要模型确定, 我们均可以求得每一颗星象的 $(\overline{\mathrm{O}-\mathrm{C}})_{x},(\overline{\mathrm{O}-\mathrm{C}})_{y}$ 及其标准差 $\left(\sigma_{x}, \sigma_{y}\right)$. 实验发现, $\mathrm{CCD}$ 支架旋转 $90^{\circ}$ 前或旋转 $90^{\circ}$ 后的各自图像集合求解出 的 $(\overline{\mathrm{O}-\mathrm{C}})_{x},(\overline{\mathrm{O}-\mathrm{C}})_{y}$ 都有很好的内部符合, 即标准差 $\left(\sigma_{x}, \sigma_{v}\right)$ 都非常小. 对于亮星, 精度平均可达 5 7 mas. 这一精度与目前国际上 $\mathrm{CCD}$ 测量精度是一致 的 ${ }^{[15]}$, 而且与我们采用的模型的阶数关系不大(图 2).

然而, 在低阶常数模型下, 当比较同一颗星旋转 前后的 $(\overline{\mathrm{O}-\mathrm{C}})$ 时, 它们具有明显的系统偏差. 图 3 显 示了 4 常数模型求解时, 旋转前平均 $(\mathrm{O}-\mathrm{C})$ 与同一 颗星旋转后平均 $(\mathrm{O}-\mathrm{C})$ 之差在赤经 (与 $x$ 轴平行)、赤 纬方向的偏差矢量图. 我们可以清楚地看到, 多数星 的偏差矢量的大小约为 100 mas. 带有漩浴状的系统 偏差说明 4 常数模型是非常不准确的. 类似的情况存 在于 6 常数模型的求解中 (见图 4), 虽然图 4 中的矢 量大小总体上有所减小, 尤其是右半部分的矢量大
小明显变小. 随着模型阶数的增加, 这种系统偏差的 大小越来越小. 图 5 和 6 分别显示了在 20 和 30 常数 模型求解的情况. 在 20 常数模型求解的图 5 矢量分 布图中, 靠近视场边缘仍然有个别星具有较明显的 偏差. 注意到图 6 中系统偏差已经非常小, 最大偏差 不到 $0 .{ }^{\prime \prime} 03$ (在赤纬方向, 且为 $m=16.01$ 的暗星, 参考 图 6 中的星 A), 而且几乎所有矢量均呈随机分布, 看 不出有系统的变化趋势. 我们也对高达量度坐标 $(x, y) 5$ 次方项的 42 常数模型进行了求解, 发现经常会 出现发散的情况. 这也从反面说明 30 常数模型是最 好的模型.

对于图 3 中漩涡状分布的矢量图, 我们似乎可以 认为主要是由量度坐标系 $x$ 和 $y$ 两个方向有不同的比 例尺造成的. 但仔细分析后发现, 这样的解释有些牵 强附会. 一方面, 如果主要是由于比例尺的不同造成, 则 6 常数模型就能基本上描述视场中的扭曲. 但图 4 中左半部分仍然具有相当大的矢量分布, 让我们感 到难以信服. 另一方面, 当我们对比 HST 视场扭曲 求解 ${ }^{[2]}$ 的图 4 发现, 这里的图 3 并没有相同之处. 也
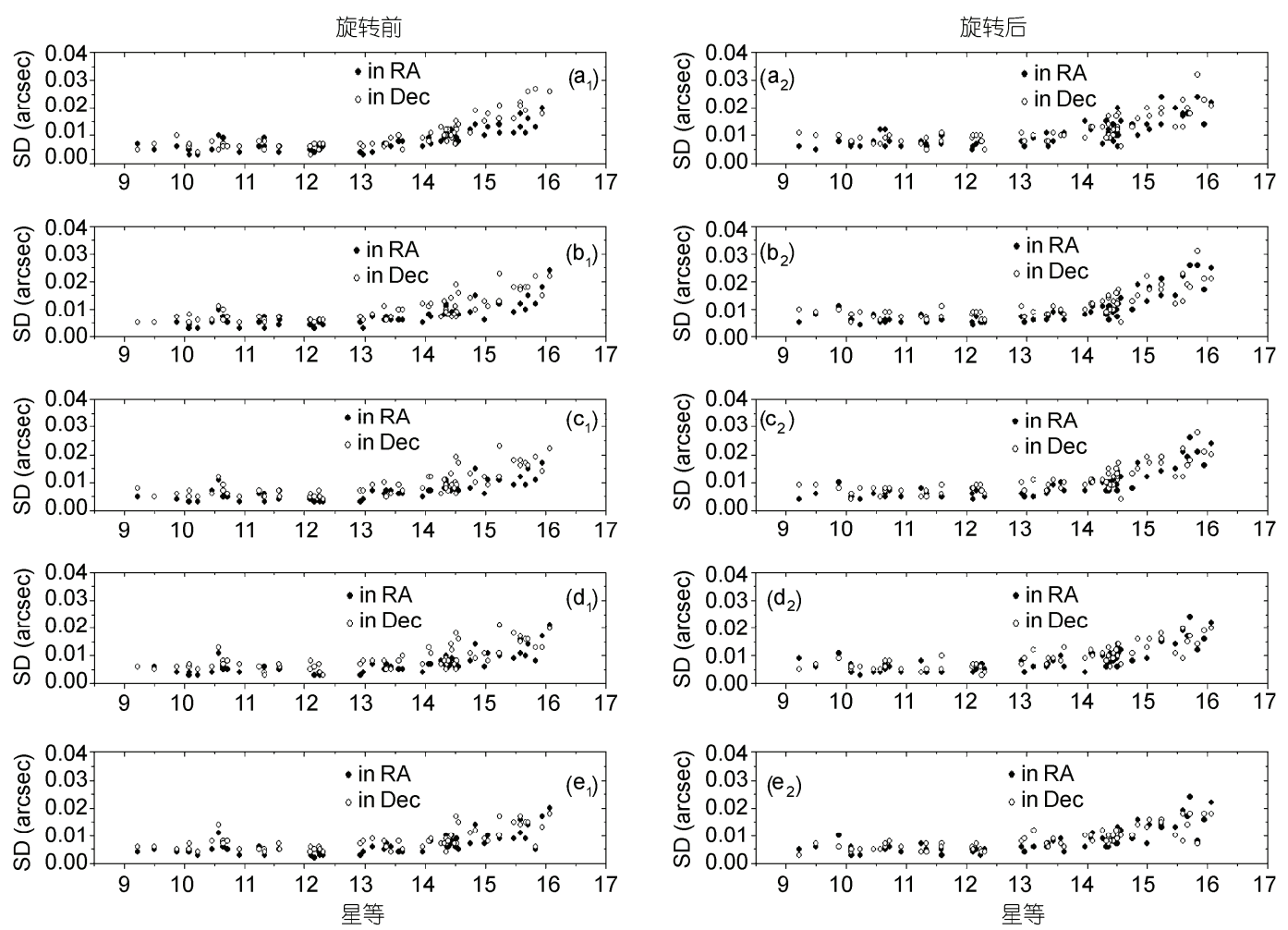

图 $2 \mathrm{CCD}$ 支架旋转 $90^{\circ}$ 前后, UCAC2 星求解的标准差

(a) (e)分别对应于 4, 6, 12, 20 和 30 常数模型求解的标准差情况, 下标 1 (如 $\mathrm{a}_{1}$ ) 和 2 分别表示 CCD 支架旋转 $90^{\circ}$ 前后的情况 


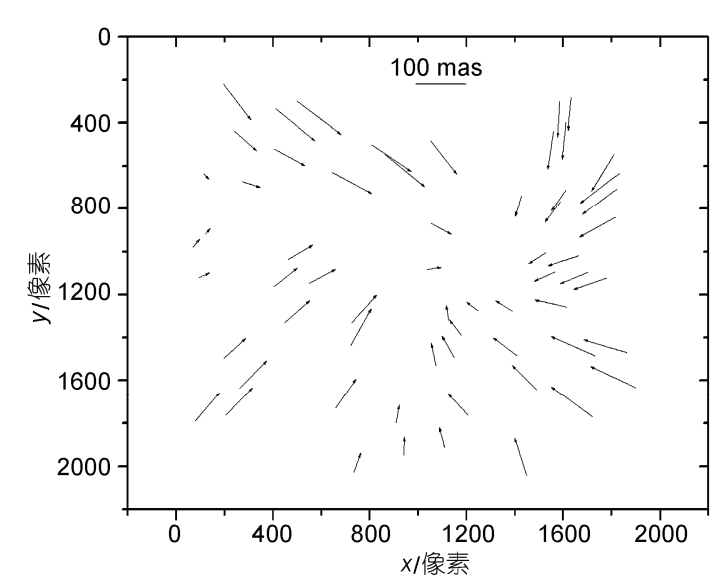

图 34 常数模型求解时, CCD 支架旋转 $90^{\circ}$ 前后平均 $(\mathrm{O}-\mathrm{C})$ 偏差的矢量分布图

矢量的起点为首幅 $\mathrm{CCD}$ 图像中星象的位置, 其余图类同

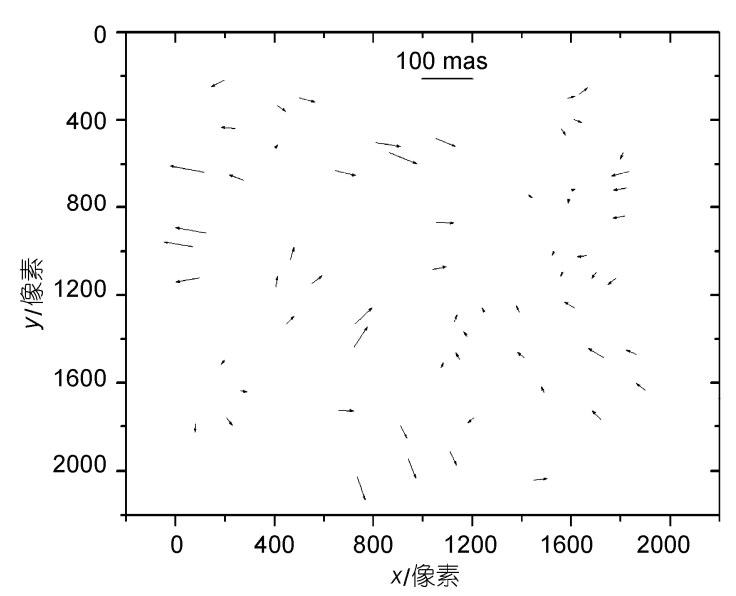

图 46 常数模型求解时, $\mathrm{CCD}$ 支架旋转 $90^{\circ}$ 前后平均 $(\mathrm{O}-\mathrm{C})$ 偏差的矢量分布图

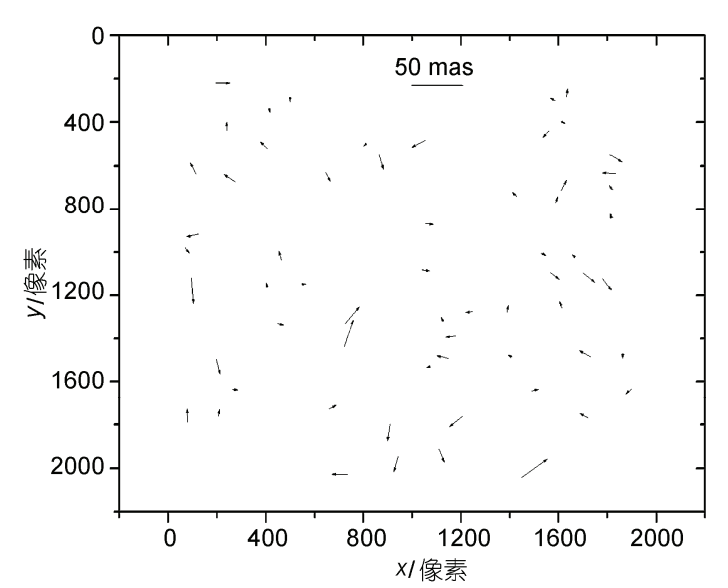

图 520 常数模型求解时, $\mathrm{CCD}$ 支架旋转 $90^{\circ}$ 前后平均 $(\mathrm{O}-\mathrm{C})$ 偏差的矢量分布图

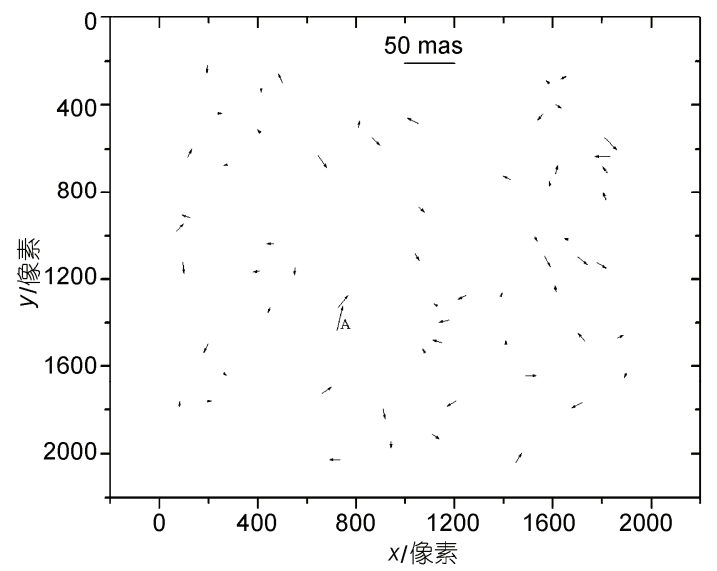

图 630 常数模型求解时, $\mathrm{CCD}$ 支架旋转 $90^{\circ}$ 前后平均 $(\mathrm{O}-\mathrm{C})$ 偏差的矢量分布图

$\mathrm{A}$ 为暗星 $(16.01 \mathrm{mag})$

许, 这里的漩浴状分布主要由高阶扭曲项的忽视而 造成. 更细致的分析有待进一步的实验研究.

作为验证, 我们还计算了 20 常数、30 常数模型 分别求解所有 27 帧 $\mathrm{CCD}$ 图像后得到的 $(\overline{\mathrm{O}-\mathrm{C}})_{x}$, $(\overline{\mathrm{O}-\mathrm{C}})_{y}$ 以及相应的标准差 $\left(\sigma_{x}, \sigma_{y}\right)$ 与星等 (取自 $\mathrm{UCAC} 2$ 星表)的关系, 如图 7 所示. 可以看到, 用 30 常数模型求解得到的标准差明显小于 20 常数模型对 应的标准差, 而且亮于 12.5 等的星, 用 30 常数模型 求解的平均标准差在每个方向约为 7 mas. 最后, 从 图 7 左列我们还发现亮于 12.5 等的星, 它们的平均 $(\mathrm{O}-\mathrm{C})$ 在赤经、赤纬两个方向都比较小, 其弥散约为 $0 .{ }^{\prime \prime} 03$, 而其余暗星的弥散就明显要大得多. 这一结 果与 $\mathrm{UCAC} 2$ 星表 ${ }^{[13]}$ 的误差特性也是吻合的.

\section{4 讨论}

人们常常认为小视场 (如 $\left.10^{\prime} \times 10^{\prime}\right)$ 内的 CCD 图像 不存在明显扭曲, 正如我们引言所述土星卫星 $\mathrm{CCD}$ 位置的归算。但我们前面的实验却表明, 即使在 $7^{\prime} \times 7^{\prime}$ 视场内, $\mathrm{CCD}$ 图像的扭曲也会非常严重! 求解的 模型需要高达量度坐标 $(x, y)$ 的 4 次方. 当考虑到哈勃 空间望远镜 WFPC2 照相机非常小的视场也存在高达 $(x, y)$ 的 3 次方的视场扭曲时, 此处更大视场 $\left(7^{\prime} \times 7^{\prime}\right)$ 的 高阶模型是不难理解的. 为什么以前没有发现这样 高阶项模型的重要原因是没有旋转 $\mathrm{CCD}$ 支架, 因此 就难以分辨出模型的优劣 (正如图 2 所显示的那样). 对此, Anderson 和 $\mathrm{King}^{[2]}$ 曾认为: 最好的方法就是去 比较相互之间具有旋转的图像. 这正是我们实验的 

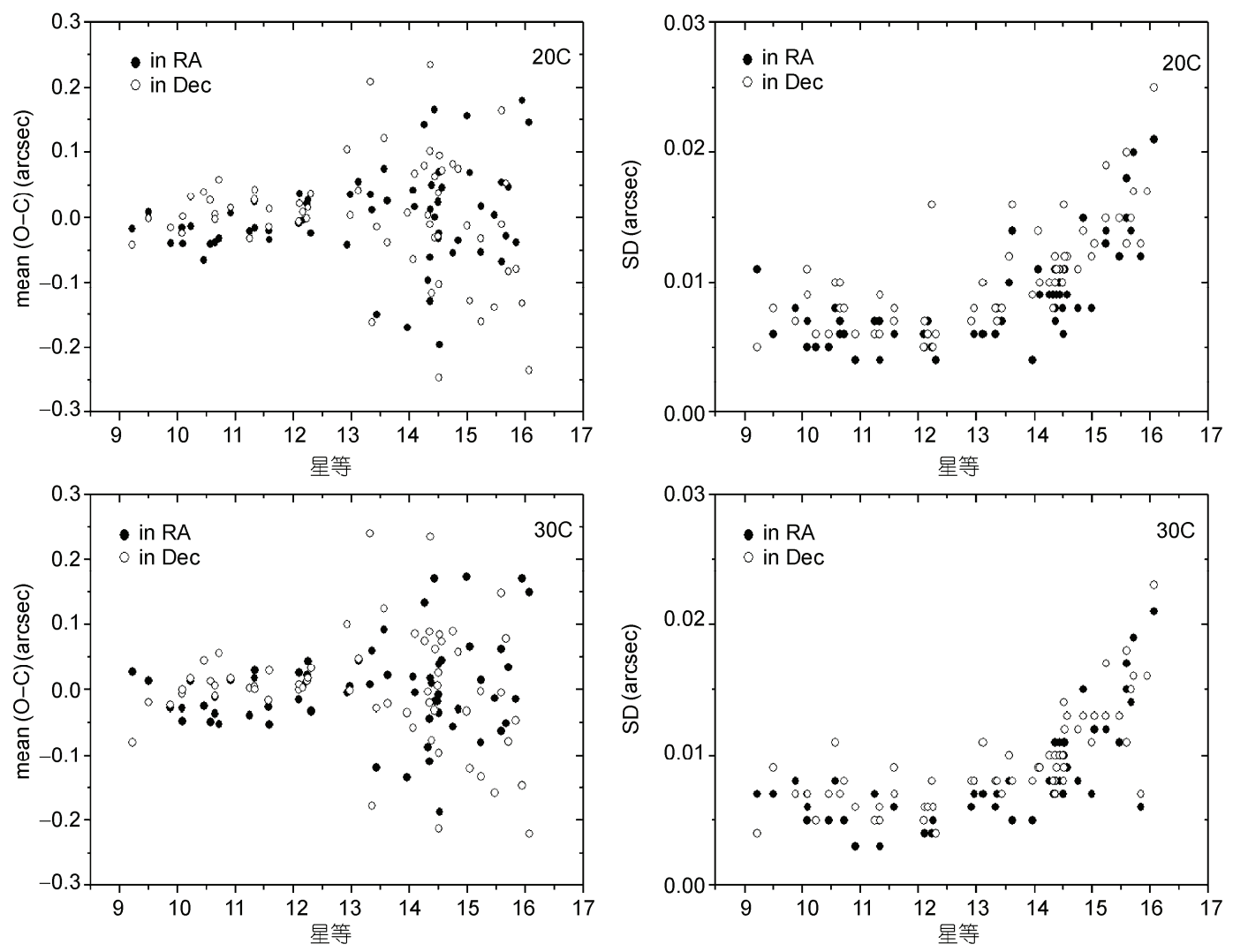

图 7 使用同一常数模型对所有图像进行归算求得每一颗星的平均 $(\mathbf{O}-\mathbf{C})$ (左列)和标准差(右列) 上面两子图表示了 20 常数模型的求解情况, 下面两子图表示了 30 常数模型求解的情况

出发点和能发现视场高阶扭曲项的关键.

在实际测量中(如观测土星或木星卫星的位置)如 何使用这种高阶模型, 这还需要深人研究. 借鉴 HST 视场扭曲求解的经验, 我们需要研究 CCD 视场在不 同取向的规律性和稳定性. 这些将留作我们进一步深 人的研究.

\section{5 结论}

使用中国科学院云南天文台 1 米望远镜上新安 装的 $2 k \times 2 k \mathrm{CCD}$ 在天顶附近观测疏散星团 NGC2168,
并旋转 $\mathrm{CCD}$ 支架 $90^{\circ}$ 前后分别成像. 星象的量度坐标 采用二维高斯函数模型进行拟合, 并使用 UCAC2 星 表进行位置精确归算表明: CCD 视场的底片常数模 型需要高达量度坐标 $(x, y)$ 的 4 次方项. 考虑到这种模 型后, UCAC2 星具有很好的内部符合和外部符合, 单次测量的位置精度在每一方向可以达到 7 mas. 这一精度对应于视场大小为 $7^{\prime} \times 7^{\prime}$ 、曝光时间为 $60 \mathrm{~s}$ 的 CCD 图像, 并与目前国际上 CCD 的最好测量精度 一致. 这种高阶底片常数模型的发现, 对 $\mathrm{CCD}$ 成像 观测用于高精度天体测量工作将具有重要的意义. 


\section{参考文献}

1 Anderson J, King I R. Toward high-precision astrometry with WFPC2. I. Deriving an accurate point-spread function. Publ Astron Soc Pac, 2000, 112: 1360-1382

2 Anderson J, King I R. An improved distortion solution for the Hubble Space Telescope's WFPC2. Publ Astron Soc Pac, 2003, 115: $113-131$

3 Poulet F, Sicardy B. Astrometric observations of inner Saturnian satellites. Astron Astrophys, 2001, 366: 339—342

4 French R G, McGhee C A, Frey M, et al. Astrometry of Saturn's satellites from the Hubble Space Telescope WFPC2. Publ Astron Soc Pac, 2006, 118: 246-259

5 Harper D, Murray C D, Beurle K, et al. CCD astrometry of Saturn's satellites 1990-1994. Astron Astrophys Suppl Ser, 1997, 121: 62-69

6 Qiao R C, Shen K X, Liu J R, et al. 1994-1996 CCD astrometric observations of Saturn's satellites and comparison with theories. Astron Astrophys Suppl Ser, 1999, 137: 1-5

7 Vienne A, Thuillot W, Veiga C H, et al. Saturnian satellite observations made in Brazil during the 1995 opposition with an astrometric analysis. Astron Astrophys, 2001, 380: 727-733

8 Peng Q Y, Vienne A, Shen K X. Positional measuring procedure and CCD observations for Saturnian satellites. Astron Astrophys, 2002, 383: $296-301$

9 Peng Q Y. New confirmation of image-processing techniques for astrometry of Saturn and its satellites. Mon Not R Astron Soc, 2005, 359: $1597-1600$

10 Peng Q Y, Vienne A, Gan L L, et al. CCD positions of Saturn and its major satellites from 2002-2006. Astron J, 2008, 136: 2214-2221

11 Bertin E, Arnouts S. SExtractor: Software for source extraction. Astron Astrophys Suppl Ser, 1996, 117: 393-404

12 李展, 彭青玉, 韩国强. CCD 图像数字定心算法的比较. 天文学报, 2009, 50: 340-348

13 Zacharias N, Urban S E, Zacharias M I, et al. The second US naval observatory CCD astrograph catalog (UCAC2). Astron J, 2004, 127: $3043-3059$

14 Tabur V. Fast algorithm for matching CCD images to a stellar catalogue. Publ Astron Soc Aust, 2007, 24: 189—198

15 Anderson J, Bedin L R, Piotto G, et al. Ground-based CCD astrometry with wide field imagers. I. Observations just a few years apart allow decontamination of field objects from members in two globular clusters. Astron Astrophys, 2006, 454: 1029—1045

・动 态・

\section{Y 形碳纳米管内的水分子信号倍增器}

分子尺度的信号变换和放大对很多物理和生物过程非 常重要，如分子开关、纳米门、生物传感器和各种神经过 程. 然而, 人们在分子水平对这些信号处理过程知之甚少, 其中部分原因是由于热波动和旁支信号干扰所带来的显著 噪声. 中国科学院上海应用物理研究所方海平研究组与合 作者，利用分子动力学模拟的方法发现被限制在狭窄 $\mathrm{Y}$ 形 纳米管道的水链可以使单电子水平的信号发生转换, 并放 大到两个或多个信号. 这种 Y 形纳米通道使分子信号发生
显著转导的能力归因于水链的强双极诱导排序, Y 形纳米 管中转换的水方向可以被主链中水方向所控制. 该系统对 电荷信号转换的反应非常迅速，在几个至几百个纳秒间. 此外, 多种水模型(TIP3P, TIP4P 和 SPC/E)模拟结果显示 在室温条件下，即使存在分支信号的干扰，Y 形碳纳米管 仍然具有很强的转导能力. 相关研究论文发表于 2009 年 10月 27 日 Proc Natl Acad Sci USA, 106(43): 18120-18124。

(信息来源：科学技术部《基础科学研究快讯》) 